\title{
Bilateral Superficial Cervical Plexuses Block Combined With General Anesthesia for Elective Thyroid Surgery
}

\author{
Fentahun Tarekegn ${ }^{1 *}$, Setegn Eshetie ${ }^{2}$ and Amare Hailekiros ${ }^{3}$

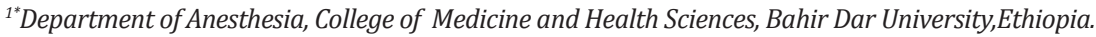 \\ ${ }^{2}$ Department of Medical Microbiology, College of Medicine and Health Sciences, University of Gondar,Ethiopia. \\ ${ }^{3}$ Department of anesthesia College of Medicine and health Science, University of Gondar,Ethiopia
}

Received: September 09, 2017; Accepted: November 04, 2017; Published: November 29, 2017

*Corresponding author: Fentahun Tarekegn, Department of Anesthesia, College of Medicine and Health Sciences, Bahir Dar University, P.o.box: 272, Ethiopia, Tel.: +251913824072; Fax number: +251584470361; E-mail: tarekegnfentahun@gmail.com

\begin{abstract}
Background: Thyroidectomy is associated with mild to moderate type of pain. The employment of regional anesthesia for thyroid surgery remains controversial for some studies. We tested the hypothesis that multiple injection of Bilateral Superficial Cervical Plexus Block (BSCPB) reduced pain scores, opioid consumption and prolongs time of analgesic request after thyroid surgery.
\end{abstract}

Methods: of 45 patients, 40 completed the study. They were allocated in to two groups: the Bilaterela Superficial Cervical Plexus Block (BSCPB) and the control group. The outcome measures were the severity of pain measured on Visual Analogue pain rating Scale (VAS), total opioid consumption, and first analgesic request time during the first postoperative 24 hours.

Results: The main outcomes recorded during the first 24 hours were Visual Analogue scale pain score (VAS, 0-10), total opioid consumption and the first analgesic request time. There were VAS scores at rest with median (IQR) in mm $9.00(5.00-16.00)$ vs. $15.00(10.00-22.00), \mathrm{p}<0.013$ and at swallowing with mean \pm SD in $\mathrm{mm} 11.00 \pm 8.52$ vs $28.70 \pm 7.40 \mathrm{p}<0.001$ for the BSCPB and control group after 24 hours of surgery respectively. It was also showed a statistically significant $(p<0.005)$ difference observations between the groups throughout the whole period of visit. Twenty four hours after surgery, total tramadol consumption was significantly reduced in cases (BSCPB) and control groups as 550 vs 2350 milligram p < 0.05 , respectively. After surgery, time for first analgesic request was significantly prolonged in BSCPB ( 560.00 vs $26.00, \mathrm{p}<0.001$ ) minutes.

Conclusion and recommendation: A multiple injection of BSCPB provided superior analgesia for elective thyroid surgery done under general anaesthesia. We recommend BSCPB to be included as part of multimodal analgesia before intubation for thyroidectomy.

Keywords: Thyroid surgery; Bilateral Superficial Cervical Plexus Block; Postoperative pain;

\section{List of abbreviation}

BSCPB: Bilateral Superficial Cervical plexus Block, VAS: Visual Analogue Scale

\section{Introduction}

Thyroid surgery causes mild to moderate strength of pain that needs to have analgesics, mostly within first day of operation. It is also believed to have discomfort on swallowing and burning sensation in the throat. These patients are tried to be managed by systemic analgesic drugs of opioids and Non Steroidal Anti Inflammatory Drugs (NSAID) in postoperative period [1]. However, both thyroid surgery and opioid type of systemic analgesics are associated with postoperative nausea and vomiting on majority of patients following thyroidectomy $[2,3,4]$.

Superficial cervical plexus covers dermatome level of second to fourth cervical nerves on anterolateral part of the neck and regional block of this plexus has been implemented to anesthetize the area of anterior triangle of the neck [5,6]. A Bilateral Superficial Cervical Plexus Nerve Block (BSCPB) is supposed to be lessening of postoperative severity of pain and reduction of postoperative analgesic consumption $[7,8]$. This type of block is the trendy regional anesthesia technique which could be done with the method of bilateral injection of local anesthetic drugs at the lateral border of sternocleidomastoid muscle to make surface anesthesia on transverse cervical, greater auricular, lesser occipital, and supraclavicular nerves [1,9].

Thyroid surgery is the most common surgical procedure in our hospital which may associate with severe postoperative pain and repeated systemic analgesic request. The BSCPB is routinely performed by senior anesthetists who have experience of greater than two years as part of multimodal analgesia in Felege Hiwot Referral Hospital, Bahir Dar, Ethiopia for Thyroid Surgery. However, its efficacy has never addressed before. In this study, we assessed the efficacy of bilateral superficial cervical plexus block with multiple injection using anatomical land mark techniques for postoperative pain control after thyroid surgery: prospective cohort study design.

\section{Methods}

The prospective cohort study design was conducted at Felege Hiwot Referral Hospital North West Ethiopia from September 1, 2016 to January 1, 2017. All consecutive thyroidectomy patients at postoperative period were included by fulfilling the inclusion criteria of ASA status I and II of either sex undergoing elective thyroid surgery and bilateral $10 \mathrm{ml}$ of $0.25 \%$ bupivacaine was 
given before intubation. There were cases rejected as exclusion criteria of abuse of drugs or alcohol, allergy to local anesthetics, refused patients, infection at site of needle injection, and bleeding disorder (data obtained from preoperative anesthetic chart record).

\section{Dependent variables}

The outcome variable from this study was postoperative pain which assessed on Visual Analogue Scale (VAS) at rest and swallowing, Total analgesic consumption, and Time of first analgesic request.

\section{Independent variables}

Sociodemographic variables (age, sex BMI, intraoperative anesthesia duration, and ASA status) were the independent variables.

\section{Operational definition}

VAS: is the method of pain assessment where patients are asked to mark their severity of pain on scale of $(0,10)$
Total analgesic consumption: is the type and amount of analgesic drugs given within 24 hours of postoperative period.

Time of first analgesic request: is the first time in which patients need analgesics at postoperative period.

\section{Sample size calculation and sampling technique}

The sample size was determined by mean comparison formula of: $n=\left(s_{1}{ }^{2}+S_{2}{ }^{2}\right) f(\alpha, \beta) \quad\left(m_{1}-m_{2}\right)^{-2}$. The data has been taken from previous study of VAS scores of cases and control group (BSCPB vs. control, mean $\pm \mathrm{SD}=(2.6 \pm 2.0)$ vs. $(5.8 \pm 1.6)$ with postoperative 24 hours duration [10]. Where, $\beta=$ beta error $=20 \%, \alpha=$ alpha error $5 \% . \mathrm{m} 1, \mathrm{~s} 1$ and $\mathrm{m} 2, \mathrm{~S} 2$ are the mean and standard deviation of case and control groups respectively. Therefore cases are 20 and controls are 20. The group of 20 cases were patients received bilateral superficial cervical plexus block (BSCPB) preoperatively (before induction of anesthesia with intubation but after given diclofenace and tramadol); the remaining group of 20 controls were patients received only systemic analgesics (Figure 1).

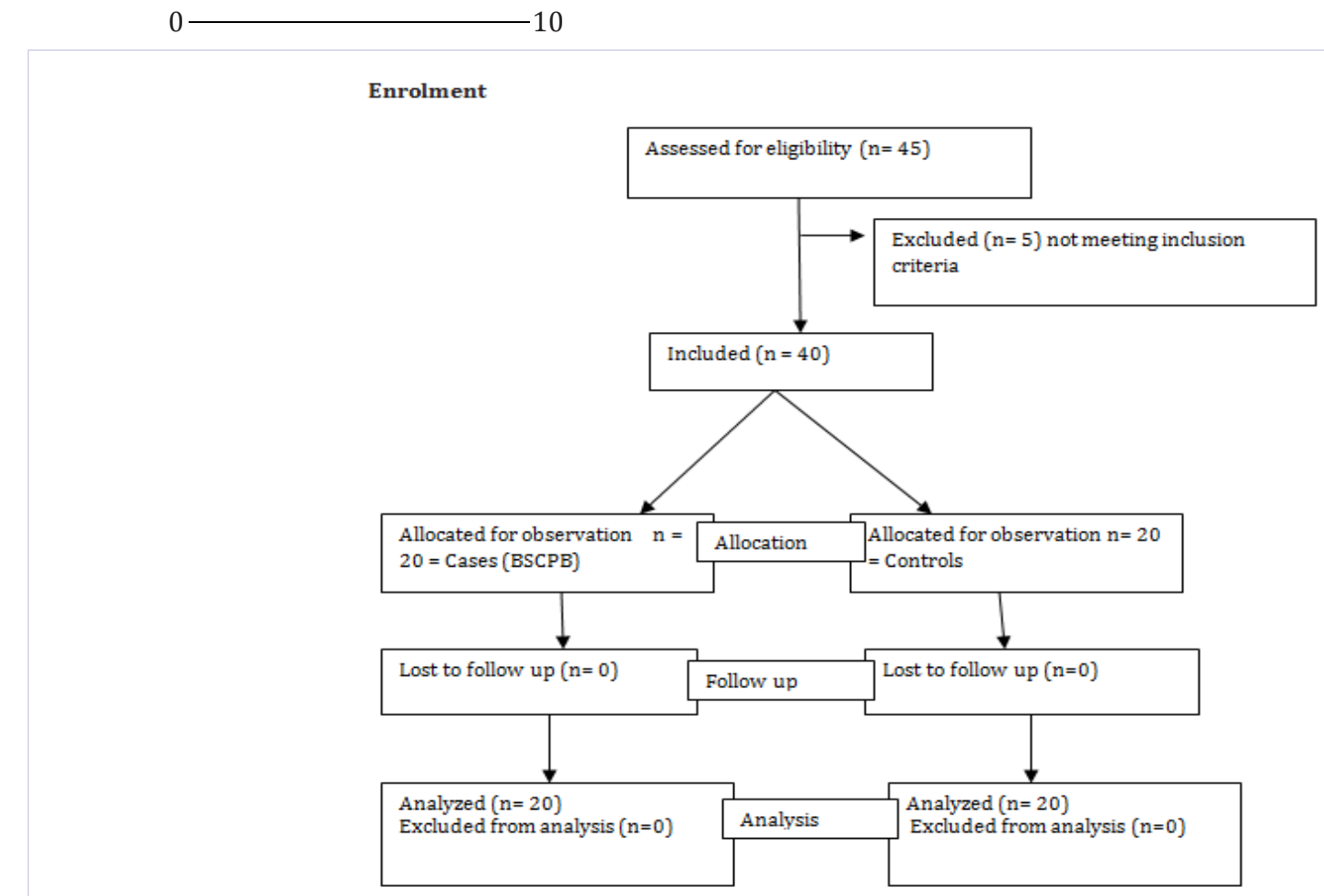

Figure 1: Consort flow diagram

The BSCPB techniques were performed by senior anesthetists' who have experience of greater than two years on both sides of the neck. The head was turned to one side after the preparation. Then $10 \mathrm{ml}$ of $0.25 \%$ bupivacaine was given at the midpoint of posterior border of sternocleidomastoid muscle by 21 gauge syringe needle. The $3.5 \mathrm{ml}$ each was injected to superiorly and inferiorly along the posterior border of sternocleidomastoid muscle through intermediate approach of fascia layer. The remaining $3 \mathrm{ml}$ was given under midpoint of this muscle after repeated aspiration.

After operation, patients were transferred to the post anesthesia care unit (PACU) then to the ward at the next day of morning. We evaluated postoperative pain with VAS on reference of $(0 \mathrm{~cm}$ : no pain to $10 \mathrm{~cm}$ : worst imaginable pain) at time of 2 hour, 4 hour, 8 hour, 12 hour ,and 24 hour after surgery, time of the first analgesic request and total analgesic consumption. The Post operative pain on swallowing was also recorded by the data collectors. 


\section{Data management and analysis}

The data were coded, cleaned, entered and analyzed by SPSS version 20 and tested for normality using Shapiro- Wilk normality test. There was normal distribution data at the time of the first analgesic request, total post operative analgesic consumption and VAS score on swallowing as checked using Shapiro-Wilk test and homogeneity of variance assessed by Levene's test for equality of variances. Therefore, an independent t-test was run on the data with $95 \%$ confident interval to analyze measurement of time of the first analgesic request and VAS score on swallowing.

However, there was not normally distributed for repeated VAS measurements at rest as checked using Shapiro-Wilk test. Therefore, non parametric Mann -Whitney U test was run on the data as well as $95 \%$ Confident Interval (CI) to analyze with further paired comparison at each time interval. The comparisons of categorical parameters were analyzed using chi-square test or Fisher's exact test. Normally distributed data are presented as mean \pm SD where as not normally distributed data presented as median (IQR) and categorical data presented by frequencies (percentages). A p value $<0.05$ was considered statistically significant.

\section{Results}

\section{Socio- demographic characteristics of the patients}

Forty patients for thyroid surgery under general anaesthesia were included in this study. Five participants were excluded from the study, two from the cases group and three from the controls group. Only 40 patients were under analysis. Of these, 20 were given Bilateral Superficial Cervical Plexus Block (BSCPB) with 10 $\mathrm{ml}$ of $0.25 \%$ bupivacaine as cases and 20 were without BSCPB block but managed by standard postoperative systemic analgesics considered as controls.

Demographic characteristics are comparable as described on (Table 1). There were no observed complications of hematoma and nerve injury.

\section{Postoperative pain VAS scores at rest}

There was reduction of VAS scores within the first 24 hours after bilateral superficial cervical plexus nerve block group compared with the control group.VAS scores as median (IQR) as shown in (Table 2)

Table 1: Demographic characteristics of patients who underwent thyroidectomy in the period of September, 2016 to January, 2017

\begin{tabular}{|c|c|c|c|}
\hline & Cases & Controls & $P$ value \\
\hline Age & $37.55 \pm 10.32^{*}$ & $43.25 \pm 16.69^{*}$ & 0.2 \\
\hline BMI & $21.84 \pm 3.25^{*}$ & $20.12 \pm 2.23^{*}$ & 0.14 \\
\hline $\begin{array}{l}\text { Intra operative duration of } \\
\text { anaesthesia in minutes }\end{array}$ & $101.50 \pm 21.09 *$ & $108.00 \pm 24.27^{*}$ & 0.12 \\
\hline \multicolumn{4}{|l|}{ Sex } \\
\hline Male & $4 / 20=20 \%$ & $7 / 20=35 \%$ & 0.91 \\
\hline Female & $16 / 20=80 \%$ & $13 / 20=65 \%$ & \\
\hline \multicolumn{4}{|l|}{ ASA status } \\
\hline \multirow[t]{2}{*}{ I } & $16 / 20=80 \%$ & $17 / 20=85 \%$ & \\
\hline & & & 0.1 \\
\hline II & $4 / 20=20 \%$ & $3 / 20=15 \%$ & \\
\hline
\end{tabular}

Note: data presented in mean \pm SD or $n(\%)$

Abbreviation: ASA, American Society of Anesthesiologists; BMI, Body Mass index

Table 2: VAS score of the patients at rest who underwent thyroid surgery in the first 24 postoperative hours in the period of September 2016 to January 2017

\begin{tabular}{|l|l|l|l|}
\hline Variables & Case = (20) & Control = (20) & P value \\
\hline VAS scores at 2 hour & $0(0)$ & $42.00(42-50)$ & $<0.001$ \\
\hline VAS scores at 4 hours & $0(0)$ & $40.50(36.00-52.00)$ & $<0.001$ \\
\hline VAS scores at 8 hour & $0(0-10.00)$ & $26.50(20.00-30.00)$ & $27.00(20.00-30.00)$ \\
\hline VAS score at 12 hour & $0(0-17.50)$ & $15.00(10.00-22.00)$ & $<0.001$ \\
\hline VAS score at 24 hour & $0(0-11.00)$ & & $<0.005$ \\
\hline Category reference $=$ median (IQR) & & \\
\hline
\end{tabular}




\section{Postoperative pain VAS scores on swallowing}

There was also significant reduction of severity of pain after Bilateral Superficial Cervical Plexus Nerve Block (BSCPB) on swallowing as shown in table (Table 3)

\section{Postoperative analgesics consumption}

The total analgesic tramadol and diclofenace consumption within 24 hours was reduced in Cases (BSCPB) group as shown in figure 2.

\section{Postoperative time for the first analgesic request}

The mean of the time for the first analgesic request was significantly prolonged in BSCPB (560.00 vs $26.00, \mathrm{p}<0.001)$ minutes.

\section{Discussion}

This prospective cohort study showed that, bilateral superficial cervical plexus block (BSCPB) through intermediate fascial layer approach performed before induction of general anesthesia with $0.25 \%$ bupivacaine, significantly reduced postoperative severity of pain on VAS, analgesic consumption, and prolonged time of the first analgesic request.

In our study, the median of VAS scores at rest were significantly reduced in cases group compared with control group during the first 24 hrs of post operative period. A comparable pattern was also showed during observation of swallowing. This could be explained that, patients with BSCPB have better-quality of analgesia than those managed with conventional systemic analgesics only. Moreover, it proven that pain has mainly superficial part after

Table 3: VAS score on swallowing of the patients who underwent thyroid surgery in the first 24 postoperative hours in the period of September 2016 to January 2017

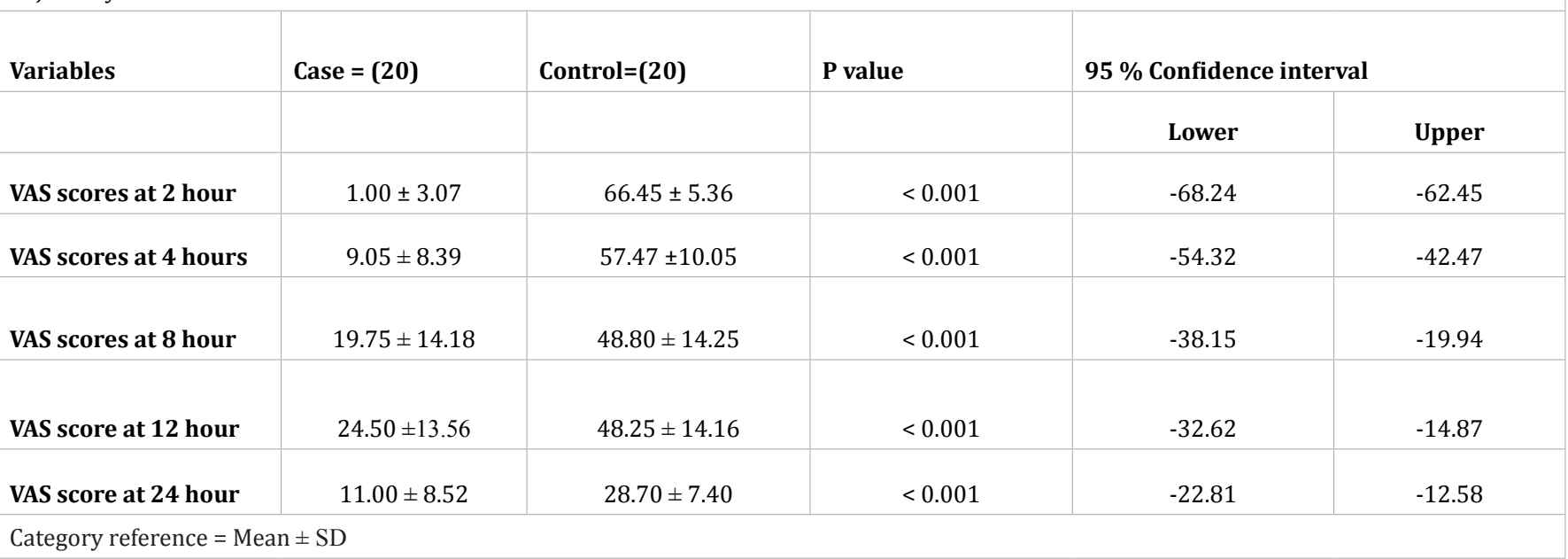

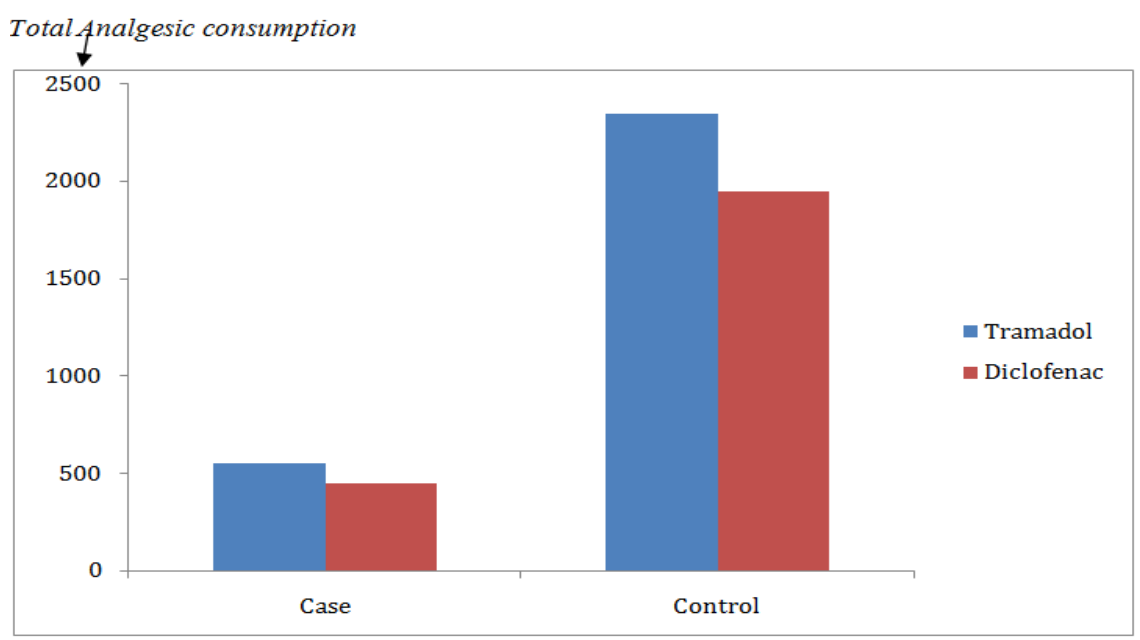

Figure 2: postoperative total tramadol and diclofenace consumption of patients who underwent thyroid surgery within 24 hours in the period of September 2016 to January 2017 
thyroidectomy even though pain perception is connected with deep layers of the cut and intra operative position of the neck $[9,11]$. It was also supported by Taiwan study which explained as the postoperative severity of pain was significantly lowered in the nerve block group [12]. A Korean study reported as bilateral superficial cervical plexus block reduced postoperative pain on swallowing and at rest with or without deep cervical plexus block done before thyroidectomy [13].

In regard to postoperative analgesic use, BSCPB group had much lower amount of postoperative tramadol consumption compared with control group. This is consistent with France study which demonstrated as all patients with regional block were not taking opiate analgesics in the first two hours of postoperative period of thyroidectomy [9]. On the other hand, postoperative diclofenace consumption was not significant in our study. This could be due to diclofenace was not administered based on WHO (World Health Organization) analgesic pain management ladder for both groups of patients.

Additionally, time of the first analgesic request was prolonged in the regional block group versus with the control group (560 vs. 26 in minutes) respectively. This result has an extensive delayed analgesic request comparing with Indian study of 4 hours (240 minutes) [11]. This might be due to the long intra operative duration of surgery reported in India, could leads the action of local anesthetics finished earlier.

Conversely, the value of BSCPB for thyroid surgery is in controversy. Herbland and his collogues didn't get analgesic effects of $0.75 \%$ ropivacaine for BSCPB given before and after operation [14]. However, they used a two point technique of nerve block which anesthetizes the main branches of superficial cervical plexus only, where as we used a three point injection technique to include block of transverse cervical branches [1].

There were no complications observed linked with bilateral superficial cervical plexus block by considering hematoma, nerve injury and infection. BSCPB is low risk approach which encouraged to be part of pain management of thyroid surgery [15].

There are some limitations to be considered in our study. The failure rate of the regional block was not checked at postoperative period. This might under estimate the quality of the block. Patients were not randomized even though they were comparable between two groups in demographic data (Table 1). Since data collectors might see the regional block procedures in the intraoperative period, It is difficult to say true blinded.

\section{Conclusion}

A multiple injection of BSCPB provided superior analgesia after Thyroid surgery combined with general anaesthesia. We recommend BSCPB to be included as part of multimodal analgesia for thyroid surgery before intubation.

\section{Acknowledgements}

We would like to thank Felege Hiwot referral Hospital anesthesia department anaesthetists for their advice and help.

\section{Declaration}

Ethics approval and consent to participate: Ethical approval was obtained from Amhara Regional Health Bureau Research Ethics Review Committee (RERC) with official permission letter to conduct the research and informed consent was obtained from each patient at preoperative period

\section{Authors' contributions}

FT, conceived the study and developed the proposal, collected the data, analyzed the data and manuscript preparation. SE, revised the proposal and involved in data collection, data analysis and manuscript preparation. $\mathrm{AH}$, revised the proposal and involved in data collection, data analysis and manuscript preparation. All authors approved the final manuscript and agreed to publication in journal of SOJ anesthesiology and pain and mangement.

\section{References}

1. Andrieu G, Amrouni H, Robin E, Carnaille B, Wattier JM, Pattou F, et al. Analgesic efficacy of bilateral superficial cervical plexus block administered before thyroid surgery under general anaesthesia. British journal of anaesthesia. 2007;99(4):561-566. doi: 10.1093/bja/ aem230

2. Sonner JM, Hynson JM, Clark O, Katz JA. Nausea and vomiting following thyroid and parathyroid surgery. Journal of clinical anesthesia. 1997; $(5): 398-402$.

3. Motamed C, Merle JC, Yakhou L, Combes X, Dumerat M, Vodinh J, et al. Intraoperative iv morphine reduces pain scores and length of stay in the post anaesthetic care unit after thyroidectomy. British journal of anaesthesia. 2004;93(2):306-307. doi: 10.1093/bja/aeh583

4. Karamanlioglu B, Turan A, Memis D, Kaya G, Ozata S, Ture M. Infiltration with ropivacaine plus lornoxicam reduces postoperative pain and opioid consumption. Canadian Journal of Anesthesia. 2005;52(10):1047-1053.

5. Standing S. The Anatomical Basis of Clinical Practice. 40th ed. Collins P, Wigley C (editors). London, UK: Churchill Livingstone Elsevier; 2008.

6. Mukhopadhyay S, Niyogi M, Dutta M, Ray R, Gayen GC, Mukherjee M, et al. Bilateral superficial cervical plexus block with or without lowdose intravenous ketamine analgesia: effective, simple, safe, and cheap alternative to conventional general anesthesia for selected neck surgeries. Local Reg Anesth. 2012;5:1-7.

7. Bagul A, Taha R, Metcalfe MS, Brook NR, Nicholson ML. Pre-incision infiltration of local anesthetic reduces postoperative pain with no effects on bruising and wound cosmesis after thyroid surgery. Thyroid. 2005;15(11):1245-1248. doi: 10.1089/thy.2005.15.1245

8. Aunac S, Carlier M, Singelyn F, De Kock M. The analgesic efficacy of bilateral combined superficial and deep cervical plexus block administered before thyroid surgery under general anesthesia. Anesthesia \& Analgesia. 2002;95(3):746-750. 
9. Dieudonne N, Gomola A, Bonnichon P, Ozier YM. Prevention of postoperative pain after thyroid surgery: a double-blind randomized study of bilateral superficial cervical plexus blocks. Anesthesia \& Analgesia. 2001;92(6):1538-1542.

10. Messner M, Albrecht S, Lang W, Sittl R, Dinkel M. The superficial cervical plexus block for postoperative pain therapy in carotid artery surgery. A prospective randomised controlled trial. European journal of vascular and endovascular surgery. 2007;33(1):50-54. doi: 10.1016/j.ejvs.2006.06.024

11. Kale S, Aggarwal S, Shastri V, Chintamani. Evaluation of the Analgesic Effect of Bilateral Superficial Cervical Plexus Block for Thyroid Surgery: A Comparison of Presurgical with Postsurgical Block. Indian Journal of Surgery. 2015;77(Suppl 3):1196-1200. doi: 10.1007/s12262-0151244-5

12. Ming-Lang Shih, Quan-Yang Duh, Chung-Bao Hsieh, Yao-Chi Liu, Chueng-He Lu, Chih-Shung Wong, et al. Bilateral Superficial Cervical Plexus Block Combined with General Anesthesia Administered in Thyroid Operations. World J Surg. 2010;34(10):2338-2343. doi: 10.1007/s00268-010-0698-7
13. Suh YJ, Kim YS, In JH, Joo JD, Jeon YS, Kim HK. Comparison of analgesic efficacy between bilateral superficial and combined (superficial and deep) cervical plexus block administered before thyroid surgery. Eur J Anaesthesiol. 2009;26(12):1043-1047. doi: 10.1097/ EJA.0b013e32832d6913

14. Herbland A, Cantini O, Reynier P, Valat P, Jougon J, Arimone Y, et al. The bilateral superficial cervical plexus block with $0.75 \%$ ropivacaine administered before or after surgery does not prevent postoperative pain after total thyroidectomy. Reg Anesth Pain Med. 2006;31:34-39.

15. Karthikeyan VS, Sistla SC, Badhe AS, Mahalakshmy T, Rajkumar N, Ali SM, et al. Randomized controlled trial on the efficacy of bilateral superficial cervical plexus block in thyroidectomy. Pain Practice. 2013;13(7):539-546. doi: 10.1111/papr.12022 